\title{
A nylon net filter coated with ethanolamine groups traps micrometastatic cancer cells with high specificity
}

\author{
MASAHIRO NODA and HISASHI HISATOMI \\ Laboratory of Cellular and Molecular Biochemistry, Department of Materials \\ and Life Science, Seikei University, Tokyo 180-8633, Japan
}

Received August 20, 2008; Accepted September 24, 2008

DOI: 10.3892/mmr_00000057

\begin{abstract}
Techniques for cancer cell separation and retrieval from peripheral blood are not only used to prevent cancer metastasis, but also to understand its underlying mechanisms. We developed a surface-modified membrane with high specificity for cancer cells. Ethanolamine groups were introduced to the surface of the nylon net filter, and human colon cancer cell line Colo201 cells were suspended in peripheral blood derived from healthy volunteers. Subsequently, these blood samples were transmitted through 20 surface-modified nylon filters. The number of cancer and blood cells in the blood samples was calculated using flow cytometry before and after filtering. Nylon net filters coated with ethanolamine groups trapped approximately $90 \%$ of the Colo201 cells, while unmodified nylon net filters were unable to trap them. On the other hand, no significant differences were observed in the filtration rate of hematocyte components between the unmodified nylon net filter and the nylon net filter coated with ethanolamine groups. These data indicate that nylon net filters coated with ethanolamine groups are able to trap Colo201 cells at a higher specificity than unmodified nylon net filters, effectively isolating and removing them from the blood, and suggest the potential benefits of the prevention of metastasis, cancer assessment and examination technologies as essential pre-treatment strategies.
\end{abstract}

\section{Introduction}

The prevention of metastasis is the single most important aim of cancer therapy. Metastasis of the primary cancer begins

Correspondence to: Dr Hisashi Hisatomi, Laboratory of Cellular and Molecular Biochemistry, Department of Materials and Life Science, Seikei University, 3-3-1 Kichijoji Kita-machi, Musashinoshi, Tokyo 180-8633, Japan

E-mail: hisatomi@st.seikei.ac.jp

Abbreviations: GMA, methacrylic acid glycidyl ester; ATR-FTIR, attenuated total reflection fourier transform infrared; GAPDH, glyceraldehyde-3-phosphate dehydrogenase

Key words: cell filtration, metastasis, surface modification when cancer cells migrate from the primary cancer via the peripheral circulation and are transported to the affected organ (1). Consequently, techniques for cancer cell separation and retrieval from peripheral blood are used not only to prevent cancer metastasis, but also to understand its underlying mechanisms. These techniques have various potential applications, such as in the clinical assessment of cancer.

Currently used techniques include the isolation of cancer cells from within the blood, magnetic bead separation (2-5) and density-gradient centrifugation (6-9); however, these are subject to problems associated with cost and operability. With regard to clinical applications, great demands are placed on devices that can be sterilized. Sterilization techniques following cancer cell isolation are complex; returning blood to the body is not ideal from an infection prevention standpoint. As a result, cancer cell isolation from blood using existing technology should primarily be used for clinical assessment (10). However, we believe that removing cancer cells from blood and then returning the blood back to a patient can lead to the effective prevention of cancer metastasis. Consequently, it is necessary to develop a low-cost polymer membrane that can be used for straightforward cellular separation. To ensure that high-polymer materials can easily be sterilized, a high level of safety must be assured. Moreover, to ensure easy access to a low-cost high-volume supply, a highly convenient disposable medical membrane device is needed. In our research, the surface modification of a porous high-polymer membrane was used to develop a cancer cell isolation membrane device.

\section{Materials and methods}

Introduction of functional groups to the membrane surface and membrane separation of tumor cells in the blood. A nylon net filter (Millipore, MA, USA) with a pore diameter of $11 \mu \mathrm{m}$ was exposed to Ar plasma (0.5 Torr, 2 min). Subsequently, methacrylic acid glycidyl ester (GMA) gas was introduced to the membrane surface for 120 min, grafting the GMA (Fig. 1). The grafted GMA was verified using the attenuated total reflection fourier transform infrared (ATR-FTIR) spectrum (Miracle $^{\mathrm{TM}}$ Single Reflection HATR; Pike Technology, WI, USA). Subsequently, it was immersed in a 50\% diethylamine solution at $30^{\circ} \mathrm{C}$ for $3 \mathrm{~h}$, and ethanolamine groups were introduced to the epoxy group at the end of the GMA $(11,12)$.

The human Colo201 colon carcinoma cell line (diameter, 12-18 $\mu \mathrm{m}$ ) was obtained from the Japan Health Science 
Table I. Membrane separation of cancer cells in peripheral blood.

\begin{tabular}{|c|c|c|c|}
\hline & Unmodified nylon net filter & Nylon net filter coated with the ethanolamine group & P-value \\
\hline Samples & 6 & 4 & \\
\hline Colo201 cells (\%) & $83.7 \quad(11.6)$ & $11.3 \quad(2.2)$ & $<0.005$ \\
\hline Red blood cells (\%) & $88.2 \quad(8.3)$ & $90.4 \quad(15.2)$ & NS \\
\hline Platelets (\%) & $93.7 \quad(17.6)$ & $89.0 \quad(17.1)$ & NS \\
\hline Lymphocytes (\%) & $85.8 \quad(12.3)$ & $68.6 \quad(8.3)$ & NS \\
\hline Monocytes (\%) & $91.2 \quad(15.8)$ & $72.6 \quad(8.9)$ & NS \\
\hline
\end{tabular}

P-value, significance determined using Fisher's exact test. NS, not significant; parentheses, not significant.

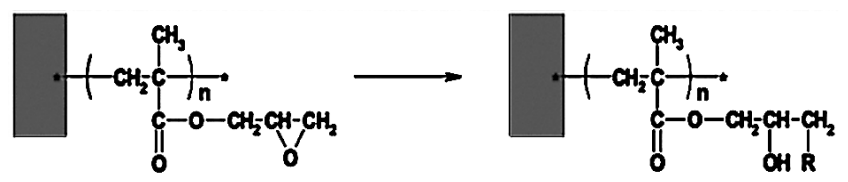

Figure 1. The anticipated product of the ring-opening reaction of the epoxy group on GMA-grafted nylon net filters.

Foundation (Osaka, Japan) and grown at $37^{\circ} \mathrm{C}$ in RPMI-1640 medium containing $10 \%$ fetal bovine serum (Gibco, NY, USA). Cells were subcultured from a 70-80\% confluent $25-\mathrm{cm}^{2}$ flask. CellTracker ${ }^{\mathrm{TM}}$ Orange (Beckman Coulter, CA, USA) was used to fluorescently label Colo201 cells, which were suspended in peripheral blood derived from healthy volunteers ( $1.5 \times 10^{4}$ Colo201 cells $\left./ \mathrm{ml}\right)$. Subsequently, $6 \mathrm{ml}$ of blood was transmitted through 20 surface-modified nylon filters at a rate of $1 \mathrm{ml} / \mathrm{min}$. The number of cancer and blood cells in the blood samples was calculated before and after filtering using an EPICS XL flow cytometer (Beckman Coulter).

Detection of CEA mRNA following membrane separation. In the peripheral blood of healthy volunteers mixed with Colo201 cells, CEA mRNAs were detected by RT-PCR as previously described (13). In brief, total RNA was extracted using Isogen reagent (Nippon Gene, Shinjuku, Japan) following the manufacturer's instructions. cDNA was synthesized with a random primer (Invitrogen, CA, USA) using $0.1 \mu \mathrm{g}$ extracted RNA. The primer set for amplification of a CEA mRNA was designed according to GenBank accession no. M29540 using primers exon 8, 5'-GGA CCT ATG CCT GTT TTG TCT-3' and exon 10, 5'-GTT GCA AAT GCT TTA AGG AAG AAG3' (230 bp). The primer set for amplification of glyceraldehyde3-phosphate dehydrogenase (GAPDH) mRNA as an internal control was designed according to GenBank accession no. M33197 using primers exon 7-8, 5'-AAC AGC CTC AAG ATC ATC AGC AA-3' and exon 9, 5'-TCA GGT CCA CCA CTG ACA CGT T-3' (313 bp). The PCR reaction was conducted at 35 cycles $\left(95^{\circ} \mathrm{C}\right.$ for $20 \mathrm{sec}, 60^{\circ} \mathrm{C}$ for $30 \mathrm{sec}, 72^{\circ} \mathrm{C}$ for $20 \mathrm{sec}$ ). The final elongation step was at $72^{\circ} \mathrm{C}$ for $5 \mathrm{~min}$. Half of the PCR product was separated using electrophoresis in a TBE buffer on $2.0 \%$ agarose gel stained with ethidium bromide and detected with ultraviolet light.

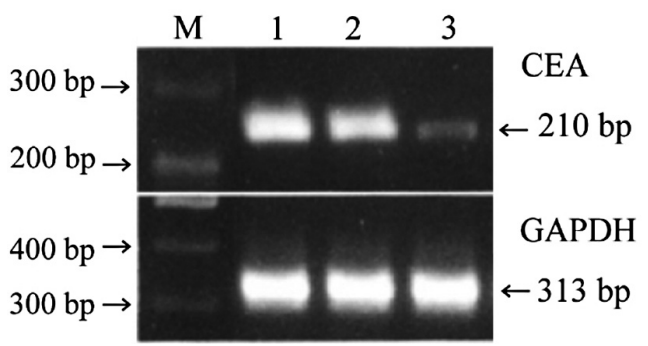

Figure 2. Detection of $C E A$ mRNA from nucleated cells following filtration through nylon net filters coated with ethanolamine groups. M, DNA size marker; 1 , after Colo201 cells were mixed into a sample of healthy human blood the nucleated cells were recovered; 2, after Colo201 cells were mixed into a sample of healthy human blood, the mixture was filtered through an unmodified nylon net filter. The nucleated cells in this filtered fluid were recovered; 3 , after Colo201 cells were mixed into a sample from healthy human blood, the mixture was filtered through a nylon net filter coated with an ethanolamine group, and the nucleated cells in this filtered fluid were recovered.

\section{Results}

Introduction of ethanolamine groups and membrane separation of tumor cells in the blood. We used the ATR-FTIR spectrum and absorbance to verify the GMA graft and introduction of ethanolamine groups.

The average transmission rate of Colo201 cells through an unmodified nylon net filter $(\mathrm{n}=6)$ was $84 \%$; $88 \%$ for red blood cells, $94 \%$ for platelets, $86 \%$ for lymphocytes and $91 \%$ for monocytes, all showing high cellular transmission rates. Conversely, nylon net filters coated with ethanolamine groups $(\mathrm{n}=4)$ trapped $\sim 90 \%$ of the Colo201 cells, while retaining the blood component transmission rates shown by the unmodified nylon net filter (Table I). Using flow cytometry, we confirmed that the shapes of filtrated blood components were maintained.

Detection of CEA mRNA. Fig. 2 shows CEA mRNA derived from nuclear cells before being filtered through the ethanolamine group-coated nylon net filter and CEA mRNA derived from nuclear cells subsequent to filtration. There was no difference in the levels of the internal control GAPDH mRNA between samples derived from cells before and after filtration. However, we confirmed that the amount of CEA mRNA was markedly decreased after blood had been filtered through the ethanolamine group-coated nylon net filter. 


\section{Discussion}

We used a polymer-based nylon net filter (nylon 66) with a pore diameter of $11 \mu \mathrm{m}$. The unmodified filter had a high filtration rate, exceeding $80 \%$ for Colo201 cells as well as most blood cell components. The relatively simple pore construction of the membrane pore diameter $(11 \mu \mathrm{m})$ of the nylon net filter used in our research exerted a minimal effect on the filtration of deformed cells, which led us to infer that supplementation is complicated. Compared with the unmodified nylon net filter, the surface of the nylon net filter coated with ethanolamine groups showed similar satisfactory filtration of blood cell components. Furthermore, the filtration rate of Colo201 cells was markedly decreased. This indicates that nylon net filters coated with ethanolamine groups were able to trap Colo201 cells with higher specificity than unmodified nylon net filters, and to effectively isolate and remove them from the blood. Moreover, in similar blood filtration experiments, the human gastric cancer cell line MKN45 (diameter, 10-16 $\mu \mathrm{m}$ ), was also confirmed to have a decreased filtration rate through a nylon net filter treated with an ethanolamine group (data not shown).

We verified that a decrease in the amount of introduced ethanolamine group to a nylon net filter (for example, grafting the GMA for $60 \mathrm{~min}$ ) acted to proportionally increase the filtration rate of Colo201 cells (data not shown). When we consider that nylon net filters coated with ethanolamine groups filter almost all blood components, we can infer that such modified membranes are highly useful membrane devices for the isolation of cancer cells.

In general, the cell-separation mechanism of the membrane is fundamentally mediated by the diameter of the membrane pores and by the physiochemical interactions of the cell and material surfaces, as well as biochemical-specific interactions influencing cell adhesive capacity on the material surface $(14,15)$. The cell-separation mechanism of the surfacemodified membranes that we developed has only a small influence on the effectiveness of the filtering performed by the physical characteristics of the membranes pores, such as size and construction, based on the cancer and blood cell component filtration rates of unmodified nylon net filters. This suggests that the functional group introduced to the membrane surface plays an important role in the filtration. At the same time, when the cationic nature of the membrane surface is excessively strong, along with the selective adsorption of cancer cells, adsorption of plasma protein and activation of blood cells are expected. We verified that an increase in the amount of functional group introduced to a nylon net filter coated with a diethylamino group acted proportionally to decrease the filtration rate of platelets and monocytes (data not shown). The reason for why the ethanolamine group has a high tumor cell adsorption ability could not be fully determined from this experiment. However, we postulate that the difference in the level of protonation in the functional group with a positive electric charge influenced cell adsorption. Further experiments will be undertaken to clarify this issue.

Physiochemical interactions were inferior to the adsorption specificity of the biological interactions. However, because of the wide range of the adsorption spectrum, universal interac- tions can be expected for a wide variety of cancer cells. When using macromolecule membranes with surfaces that possess highly selective adsorptive capacity for cancer cells, it is difficult to achieve similar economic efficiency and operability as existing technology such as flow cytometry, magnetic cell isolation and density-gradient centrifugation $(10,16)$.

In the present study, we successfully demonstrated membrane separation through selective adsorption of cancer cells in blood using the surface of a macromolecule membrane treated with a suitable amount of a functional group. Removal technology, focusing on cancer cells in blood and using a macromolecule surface, offers new treatment approaches for controlling the levels of circulating cancer cells. In addition, we believe that a pre-treatment strategy using macromolecule membranes for cancer cell separation is important for the realization of accelerated cancer assessment at medical centers, simplifying the process and achieving lower costs. Thus, we anticipate its future development. In the next stage, for clinical application, an investigation of the cellular functions and changes to intracellular signals in the filtrated cells is necessary.

\section{References}

1. Wong IH: Transcriptional profiling of circulating tumor cells: Quantification and cancer progression. Oncol Rep 10: 229-235, 2003.

2. Stift A, Friedl J, Dubsky P, et al: In vivo induction of dendritic cell-mediated cytotoxicity against allogeneic pancreatic carcinoma cells. Int J Oncol 22: 651-656, 2003.

3. Nakanishi H, Ozaki T, Nakamura Y, Hashizume K, Iwanaka T and Nakagawara A: Purification of human primary neuroblastomas by magnetic beads and their in vitro culture. Oncol Rep 17: 1315-1320, 2007.

4. Balic M, Dandachi N, Hofmann G, et al: Comparison of two methods for enumerating circulating tumor cells in carcinoma patients. Cytometry B Clin Cytom 68: 25-30, 2005.

5. Meye A, Bilkenroth U, Schmidt U, et al: Isolation and enrichment of urologic tumor cells in blood samples by a semi-automated CD45 depletion autoMACS protocol. Int J Oncol 21: 521-530, 2002.

6. Blümke K, Bilkenroth U, Schmidt U, et al: Detection of circulating tumor cells from renal carcinoma patients: Experiences of a two-center study. Oncol Rep 14: 895-899, 2005.

7. Neuhof D, Bischof M, Debus J and Weber KJ: Telomerase activity in peripheral blood mononuclear cells after whole body irradiation. Oncol Rep 17: 865-869, 2007.

8. Rosenberg R, Gertler R, Friederichs J, et al: Comparison of two density gradient centrifugation systems for the enrichment of disseminated tumor cells in blood. Cytometry 49: 150-158, 2002.

9. Edwards M, Twin J and Wilkinson S: New technique to assess the axilla for breast cancer metastases using cell separation technology. ANZ J Surg 72: 655-659, 2002.

10. Zabaglo L, Ormerod MG, Parton M, Ring A, Smith IE and Dowsett M: Cell filtration-laser scanning cytometry for the characterisation of circulating breast cancer cells. Cytometry A 55: 102-108, 2003

11. Tsuneda S, Saito K, Furusaki S and Sugo T: High-throughput processing of proteins using a porous and tentacle anionexchange membrane. J Chromatogr A 689: 211-218, 1995.

12. Kim M, Kiyohara S, Konishi S, Tsuneda S, Saito K and Sugo T: Ring-opening reaction of poly-GMA chain grafted onto a porous membrane. J Membr Sci 117: 33-38, 1997.

13. Nagao K, Hisatomi H, Hirata H, et al: Expression of molecular marker genes in various types of normal tissue: Implication for detection of micrometastases. Int J Mol Med 10: 307-310, 2002.

14. Dzik S: Leukodepletion blood filters: filter design and mechanisms of leukocyte removal. Transfus Med Rev 7: 65-77, 1993.

15. Dzik S: Mechanisms of leukocyte removal by filtration. Curr Stud Hematol Blood Transfus 60: 123-133, 1994.

16. Ring AE, Zabaglo L, Ormerod MG, Smith IE and Dowsett M: Detection of circulating epithelial cells in the blood of patients with breast cancer: comparison of three techniques. Br J Cancer 92: 906-912, 2005. 
